\title{
Performance of the Brookhaven Photocathode RF Gun
}

K. Batchelor, I. Ben-Zvi, R.C. Fernow, J. Fischer, A.S Fisher, J. Gallardo, G. Ingold, H.G. Kirk, K.P. Leung, R. Malone, I. Pogorelsky,

T. Srinivasan-Rao, J. Rogers, T. Tsang, J. Sheehan, S. Ulc, M. Woodle, J. Xie, R.S. Zhang

Brookhaven National Laboratory*, Upton NY 11973

and

L.Y. Lin

Department of Applied Physics, Columbia University, New York NY 10027

and

K.T. McDonald, D.P. Russell

Department of Physics, Princeton University, Princeton NJ 08544

and

C.M. Hung

Department of Physics, SUNY at Stony Brook, Stony Brook NY 11794

and

X.J. Wang

Department of Physics, UCLA, Los Angeles CA 90024

\begin{abstract}
The Brookhaven Accelerator Test Facility (ATF) uses a photocathode if gun to provide a high-brightness electron beam intended for FEL and laser-acceleration experiments. The rf gun consists of $1 \frac{1}{2}$ cells driven at $2856 \mathrm{MHz}$ in $\pi$-mode with a maximum cathode field of $100 \mathrm{MV} / \mathrm{m}$. To achieve long lifetimes, the photocathode development concentrates on robust metals such as copper, yttrium and samarium. We illuminate these cathodes with a 10-ps, frequency-quadrupled Nd:YAG laser. We describe the initial opfiation of the gun, including measurements of transverse and iongitudinal emittance, quantum efficiencies, and peak current. These results are compared to models.
\end{abstract}

* Work supported by DOE Contract No. DE-ACO2-76-CH00016.

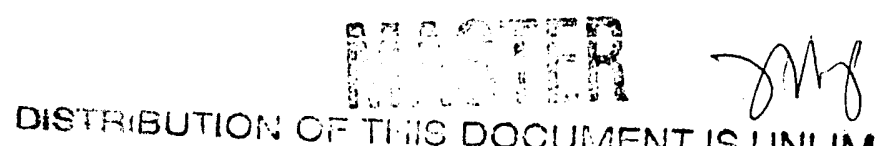




\section{Introduction}

There are several important applications in which the brightness of electron beams is a paramount consideration. Two applications which come immediately to mind are high-luminosity linear colliders and free-electron lasers.

A common requirement for these programs is the need for electron beams combining high-peak current with very small transverse and longitudinal emittances. At the Brookhaven Accelerator Test Facility we are devoting substantial resources to the production and understanding of electron beams that have these attributes.

The ultimate aim is to provide a source of electrons with as high a brightness as possible. Beam brightness is defined as the ratio of the beam peak current to the product of the horizontal and vertical normalized rms emittances:

$$
B=\frac{I_{p}}{\varepsilon_{N, x} \varepsilon_{N, y}} .
$$

We feel that the issues of producing high-brightness electron beams can be best addressed with an rf gun that contains a laser-illuminated photocathode. Our if gun program is based on the pioneering work at Stanford [1], where the first rf gun was developed, and Los Alamos [2], where a photocathode was first installed in an rf gun.

\section{Description of the BNL RF Gun}

The Brookhaven rf gun [3] is a $1 \frac{1}{2}$ cell, side-coupled structure operating at 2.856 $\mathrm{GHz}$ (see Fig. 1). A design goal is to achieve a gain in beam brightness by minimizing the emittance of the electron beam. Two effects dominate to cause growth in emittance beyond the thermal emittance of the electrons at the photocathode itsclf. These effects are the dynamics of the interaction of the electrons with the rf fields in the gun and the emittance growth caused by the self-fields of the electrons.

Emittance growth due to the rf dynamics can be controlled by ensuring that nonlinear transverse components of the rf fields are minimized. This is accomplished by placing relatively thick disks between cells and adjusting the diameter of the apertures so as to minimize nonlinear transverse components. Note that since we use a $\pi$-mode for acceleration, the rf fields are near a minimum when the particles traverse the apertures.

The strategy used to control emittance growth due to the self-fields of the electrons is to operate the gun so that the electrons are launched in a high-gradient accelerating field. Once the electrons are relativistic, emittance growth due to space charge becomes more manageable. The BNL if gun has been designed to operate with a $100-\mathrm{MV} / \mathrm{m}$ peak accelerating gradient at the cathode. Three design features ensure this: (1) the 2.856-GHz operating frequency, (2) the relatively low peak-wall field to peak-axial field ratio (1.18 to 1$)$, and (3) an initial $\frac{1}{2}$ cell to ensure that the particles are launched near peak accelerating gradients.

The rf gun has a photocathode installed on the back plane of the half cell. At Brookhaven, we are concentrating our photocathode $R \& D$ on metals, such as yttrium, samarium and copper, because metals should provide the robustness necessary for continuous operations over long periods. Copper is particularly attractive, because it allows for the possibility of constructing a simple, all-copper cavity. 
A consequence of the choice of these metals is the need for ultraviolet laser light to excite the photocathode. For copper, the 4.3-eV work function lies just below the 4.66-eV energy of frequency-quadrupled Nd:YAG $(266 \mathrm{~nm})$, and so provides a small intrinsic thermal emittance. The quantum efficiency can be $10^{-4}$. At the same wavelength, yttrium and samarium can have quantum efficiencies up to $10^{-3}$, but the 3-eV work function can lead to a higher thermal emittance. Thus, with over 100 $\mu \mathrm{J}$ of UV light available, we can extract over $10 \mathrm{nC}$ from an yttrium or samarium cathode. Our design calls for a 1-nC micropulse with a 6-ps (FWHM) pulse length, thus yielding a peak current of $160 \mathrm{~A}$. If we choose to focus onto a 3-mm-radius spot on the photocathode, this corresponds to $560 \mathrm{~A} / \mathrm{cm}^{2}$ issuing from the iurface, well within the capabilities of these materials [4].

\section{Description of the Nd:YAG laser sy stem}

The photocathode laser system is illustrated in Fig. 2, and the key parameters are listed in Table 1. A Spectra-Physics model $3400 \mathrm{CW}$ mode-locked Nd:YAG oscillator $(1064 \mathrm{~nm})$ produces a pulse train with a spacing of $12.25 \mathrm{~ns}(81.6 \mathrm{MHz})$, every $35^{\text {th }}$ rf period of the gun and linac. A Lightwave Electronics series 1000 timing stabilizer [5] reduces timing jitter of the laser pulses to 1 ps with respect to the rf. The 80 -ps oscillator pulses are frequency chirped in $200 \mathrm{~m}$ of single-mode optical fiber. A pulsed, regenerative amplifier [6] then selects, amplifies, and compresses a single pulse from the train. The selection is accomplished by a Pockels cell inside the amplifier cavity. After 12 passes through the Nd:YAG rod, the pulse is extracted by a second Pockels cell. The bandwidth of the amplifier rod is narrower than the chirp from the fiber; consequently, only the middle of the pulse is amplified and the output is expected to be compressed to $12 \mathrm{ps}$ (FWHM). Frequency quadrupling reduces the ultraviolet pulse width to $6 \mathrm{ps}$ (FWHM). Up to $300 \mu \mathrm{J}$ of ultraviolet has been incident on the cathode, focused to spot sizes ranging from 0.2 to $1.2 \mathrm{~mm}$.

Table 1 Nd:YAG Laser Parameters

\begin{tabular}{lll}
\hline & Design & Achieved \\
\hline IR Energy, mJ & 10 & 10 \\
UV Energy, $\mu \mathrm{J}$ & 50 & 300 \\
IR Pulse length (FWHM), ps & 12 & 20 \\
Pulse timing jitter, ps & $<3$ & 1 \\
Rep. Rate, Hz & 6 & 6
\end{tabular}

This system will be extended to amplify a train of 1 to 100 pulses with a spacing of 24.5 ns. This will permit operation of the ATF FEL oscillator [7], which requires a train of electron bunches rather than a single bunch. 


\section{Operation of the RF Gun}

Operation of the rf gun with the Nd:YAG laser system began in November, 1990. We have operated for an integrated run time of $\approx 700$ hours with a single copper photocathode. The of gun has been let up to air twice during this period and we find that after a subsequent bakeout, conditioning the gun to peak fields recuires $\approx 8$ hours. During normal resumption of operations without a vacuum break, reconditioning is minimal. The vacuum in the gun is typically $\approx 10^{-9}$ Torr before of power is introduced and goes to $\approx 10^{-8}$ Torr during operation of the gun.

Under special running conditions with a reduced laser spot size we have exceeded the damage threshold on copper (verified visually after cathode removal) but have not experienced any noticeable adverse effects during subsequent operation of the rf gun. This confirms the robustness of an rf gun with metal cathodes.

The peak momentum achieved to date is $4.6 \mathrm{MeV} / \mathrm{c}$, with $6.2 \mathrm{MW}$ of rf power in the gun. This output momentum corresponds to a peak-axial electric field in the rf gun of $98 \mathrm{MV} / \mathrm{m}$, which is nearly our design value of $100 \mathrm{MV} / \mathrm{m}$.

The measurement of the transverse emittance of the electron beam is done at either profile monitors 1 and 2 for the momentum-dispersed beam (see Fig. 3) or profile monitors 3 and 4 for the momentum-recombined beam. For the momentumdispersed beam, we can only measure the vertical emittance. These results, however, more nearly represent the emittance of the beam as produced by the gun, whereas measurements at monitors 3 and 4 include any growth in the transverse emittance due to the injection system.

The transverse emittance is analysed by one of two methods. The quadrupole variation method entails varying the strength of a quadrupole preceeding an individual profile monitor and observing the corresponding variations in the spot size on that monitor. Figure 4 shows an example of data collected in this manner.

An alternative method consists of focusing the beam on one profile monitor, measuring the resulting spot size of the focused beam, and then observing the beam spot on the companion monitor without readjusting the beam optics. The emittance is obtained by

$$
\epsilon_{n}=\gamma \sigma_{y}^{0} \sigma_{y^{\prime}}, \quad \text { and } \quad \sigma_{y^{\prime}}^{2}=\left(\sigma_{y}^{2}-\sigma_{y}^{02}\right) / L_{\text {drift }}^{2},
$$

where the measured quantities $\sigma_{y}^{0}$ and $\sigma_{y}$ are respectively the focused and unfocused rms profile widths; $\sigma_{y^{\prime}}$ is the inferred divergence of the beam and $L_{d r i n}$ is the known drift distance between the two profile monitor screens. Note that the roles of the two companion monitors can be reversed, thus giving two independent measurements of the emittance. The measurement also has the convenient property of being free of interpretation since knowledge of the characteristics of the quadrupoles in the beam line is not required in order to extract the emittance from the measurements.

The longitudinal structure of the electron pulse is an important beam parameter which, along with the charge, determines the peak current of the beam. This longitudinal profile, which is measured by means of an rf kicker cavity installed before the first dipole (see Fig. 3), gives the length of the electron pulses as they leave the if gun. This kicker operates at $2856 \mathrm{MHz}$ and provides a vertical displacement proportional to time, which can be observed at either the momentum slit (located between elements Q4 and Q5) or profile monitor 1. The resolution of this measurement system approaches $1 \mathrm{ps}$. 
Table 2 shows the results of the most recent measurements of the parameters of the electron beam from the if gun. Both the peak current and the beam brightness are the results of measurements taken at one occasion and are not obtained using best results for the other parameters. The $2 \mathrm{nC}$ electron beam was measured at the momentum slit when the copper photocathode was illuminated with $170 \mu \mathrm{J}$ of $266 \mathrm{~nm}$ laser light. This yields a collection efficiency (quantum efficiency $\times$ beam transport efficiency) of $0.6 \times 10^{-4}$.

Table 2 BNL RF Gun Results to Date

\begin{tabular}{lll}
\hline & Design & Achieved \\
\hline Energy, MeV & 4.6 & 4.6 \\
Repetition rate, $\mathrm{Hz}$ & 6 & 6 \\
Electron pulse charge, $\mathrm{nC}$ & 1.0 & 2.0 \\
Electron pulse length, rms, ps & 2.5 & 4.9 \\
Peak Current, A & 160 & 133 \\
Energy spread, rms, $\%$ & 0.3 & 0.4 \\
Emittance $\left(\gamma \sigma_{x} \sigma_{x^{\prime}}\right), \pi \mathrm{m}$-rad & $7 \times 10^{-6}$ & $4 \times 10^{-6}$ \\
Beam Brightness, $\mathrm{A} / \mathrm{m}^{2}$ & $3 \times 10^{12}$ & $7 \times 10^{12}$
\end{tabular}

\section{Comparison with Models}

Figure 5 shows results of measuring the energy of the electrons ejected from the if gun as a function of the input rf power. The measured shunt impedance is $50 \mathrm{M} \Omega / \mathrm{m}$ (without transit time) compared to a SUPERFISH predicted value of $57 \mathrm{M} \Omega / \mathrm{m}$.

The principal modelling tool used at BNL for studies of the beam dynamics of the rf gun is PARMELA. We show in Fig. 6 a plot of the measured electron beam momentum versus the if phase at which the laser beam strikes the photocathode. The solid line represents the PARMELA predictions.

Our measured rms emittance of $4 \pi \mathrm{mm}$-mrad is lower than the PARMELA prediction (with space charge included) by a factor of 2 . We find, however, that other codes such as MAGIC [8] tend to give results [9] similar to our observations.

With a streak camera, we have measured the amplified IR laser pulse length to be typically 20 ps (FWHM) which implies a UV pulse length of 10 ps (FWHM). This agrees well with our low-charge beam length measurement of $\sigma_{\tau}=4.9$ ps. Further measurements of a $2 \mathrm{nC}$ electron beam indicate that the pulse length has increased to $\sigma_{r}=6$ ps. PARMELA calculations give a bunch lengthening of $25 \%$ per $\mathrm{nC}$ of bunch charge in this regime. 


\section{Conclusions}

Our initial operation of the BNL rf gun has shown that our design goals for key beam parameters, including peak current and brightness, can be meet with the installation of a copper photocathode. Further, we note that the use of copper provides great mechanical and chemical stability to the gun and will allow for great simplifications in future rf gun designs.

\section{References}

1. S.V. Benson et al, Nucl. Instr. and Meth., A250 (1986), p. 39.

2. J.S. Fraser et al, Photocathodes in Accelerator Applications, Proceedings of the 1987 IEEE Particle Accelerator Conference, Washington D.C., E. Lindstrom and L.S. Taylor eds. (1987) p. 1705.

3. K. Batchelor, H. Kirk, J. Sheehan, M. Woodle and K. McDonald, Development of a High Brightness Electron Gun for the Accelerator Test Facility at Brookhaven National Laboratory, Proceedings of the 1988 European Accelerator Conference, Rome, Italy, June 2-12, 1988.

4. J. Fischer, T. Srinivasan-Rao, and T. Tsang, UV Photoemission from Metal Cathodes for Picosecond Power Switches, Proceedings of the Switched Power Workshop, Shelter Island, NY, October, 1988, R.C. Fernow, ed. BNL 52211, (1989) p. 44.

5. M.J.W. Rodwell, D.M. Bloom, and K.J. Weingarten, IEEE J. Quantum Electronics 25, (1989) p. 817.

6. T. Shimada, I. Bigio, R.F. Harrison, and N.A. Kurnit, Generation of High-Brightness $1.06 \mu \mathrm{m}$ Pulses by Compression of a Chirped Seed in a Nd:YAG Regenerative Amplifier, Conference Presentation TUJ 39, CLEO, Baltimore, April 24-28, 1989.

7. K. Batchelor, I. Ben-Zvi, R.C. Fernow, A.S. Fisher, A. Friedman, J. Gallardo, G. Ingold, H. Kirk, S. Kramer, L. Lin, J.T. Rogers, J.F. Sheehan, A. van Steenbergen, M. Woodle, J. Xie, L.H. Yu, R. Zhang, Status of the Visible Free-Electron Laser at the Brookhaven Accelerator Test Facility, Proceedings of the $13^{\text {th }}$ Int. FEL Conf., Santa Fe, NM, 26-30 Aug. 1991.

8. G.D. Warren, L. Ludeking, J. McDonald, K. Nguyen, and B. Goplen, MAGIC Users' Group Software, Proceedings of the Conference on Computer Codes and the Linear Accelerator Community, Los Alamos National Laboratory, January 22-25, 1990, Richard K. Cooper ed. LA-11857-C (1990) p. 57.

9. I.S. Lehrman, I.A. Birnbaum, S.Z. Fixler, R.L. Heuer, S. Siddiqi, E. Sheedy, K. Batchelor, I. Ben-Zvi, J. Gallardo, H. Kirk, T. Srinivasan-Rao, G.D. Warren, Design of a High-brightness, High-duty Factor Photocathode Electron Gun, Proceedings of the $13^{\text {th }}$ Int. FEL Conf., Santa Fe, NM, 26-30 Aug. 1991.

\section{DISCLAIMER}

This report was prepared as an account of work sponsored by an agency of the United States Government. Neither the United States Government nor any agency thereof, nor any of their employees, makes any warranty, express or implied, or assumes any legal liability or responsibility for the accuracy, completeness, or usefulness of any information, apparatus, profuct, or process disclosed, or represents that its use would not infringe privately owned rights. Reierence herein to any specific commercial product, process, or service by trade name, trademark, manufacturer, or otherwise does not necessarily constitute or imply its endorsement, recommendation, or favoring by the United States Government or any agency thereof. The views and opinions of authors expressed herein do not necessarily state or reflect those of the United States Government or any agency thereof. 


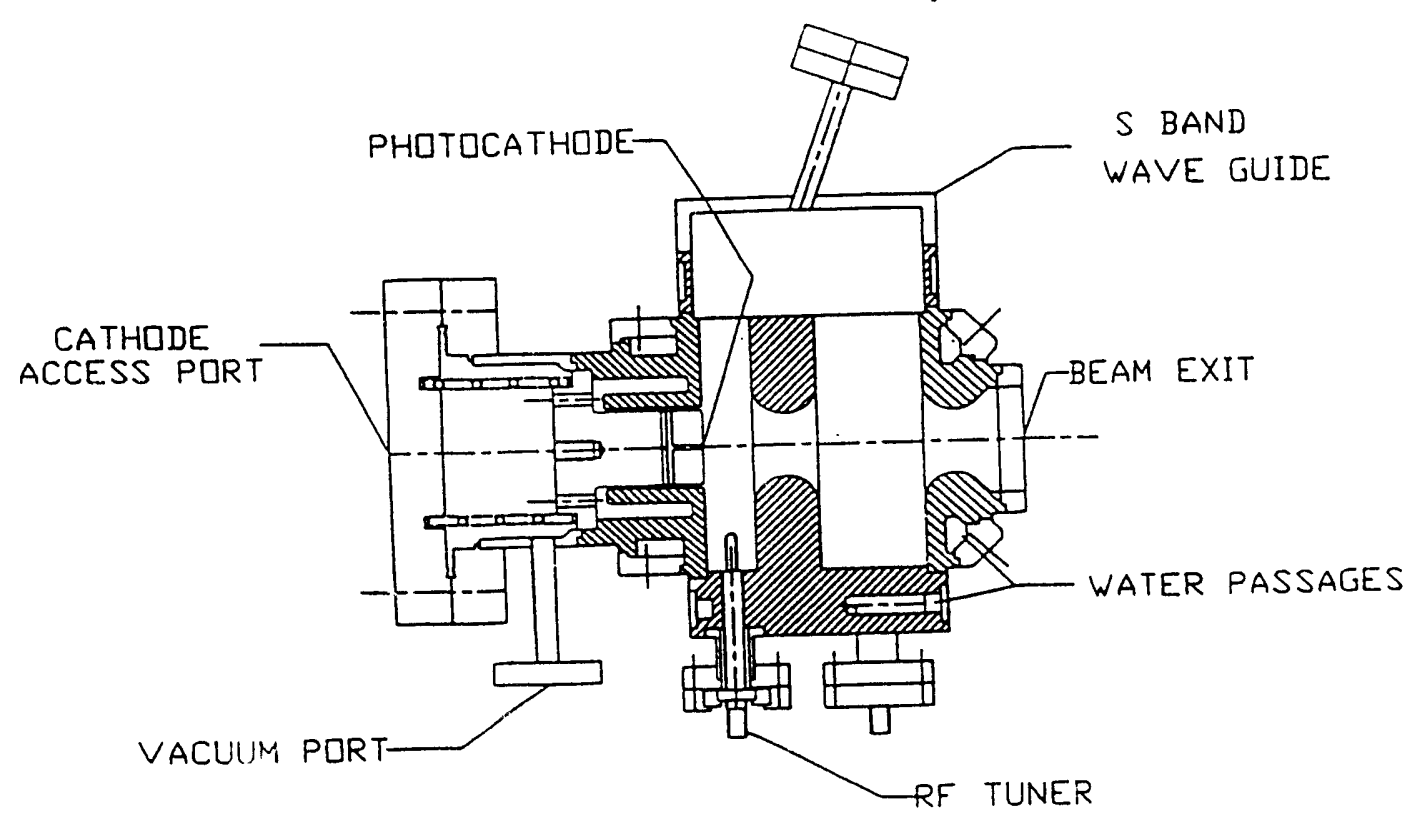

Fig. 1 The Brookhaven $1 \frac{1}{2}$ cell gun

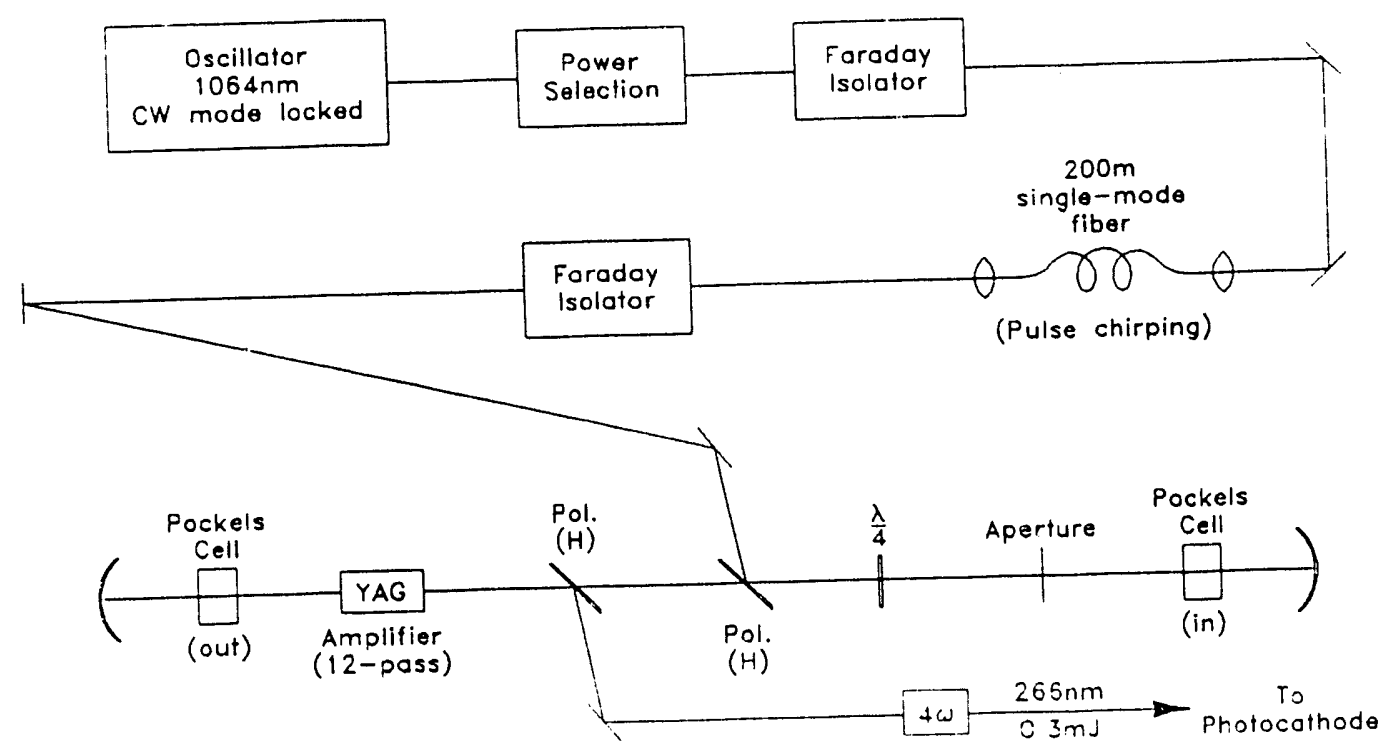

Fig. 2 Schematic of the ATF Nd:YAG laser systems 


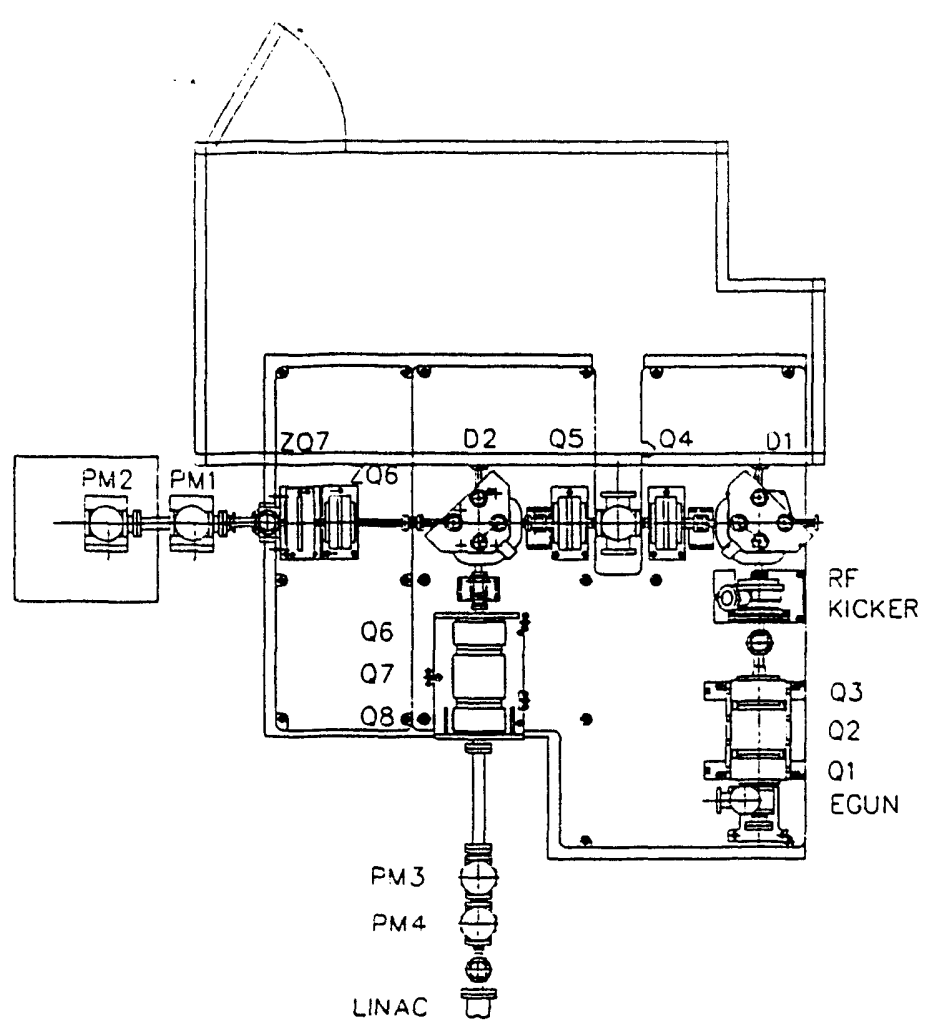

Fig. 3 The ATF injection system

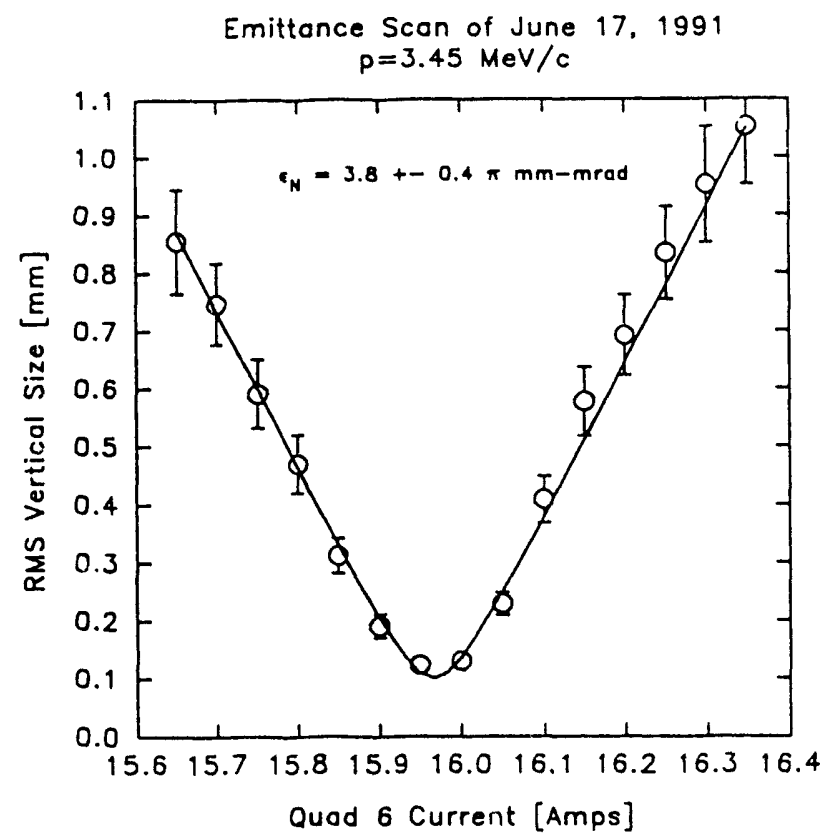

Fig. 4 The vertical projections of the electron beam on a profile monitor as a function of quadrupole current 
BNL RF Gun

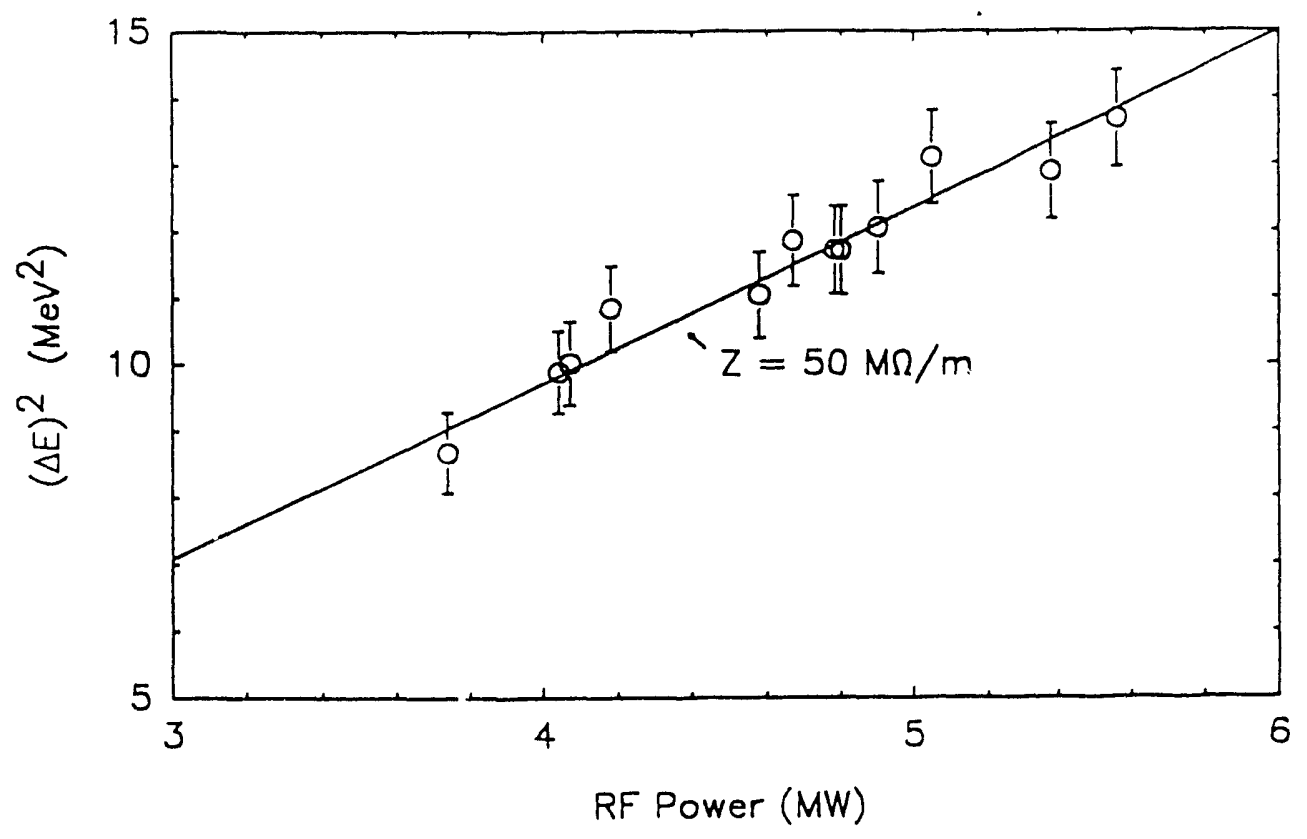

Fig. 5 The square of the energy boost to the electrons as a function of input power from the BNL RF gun

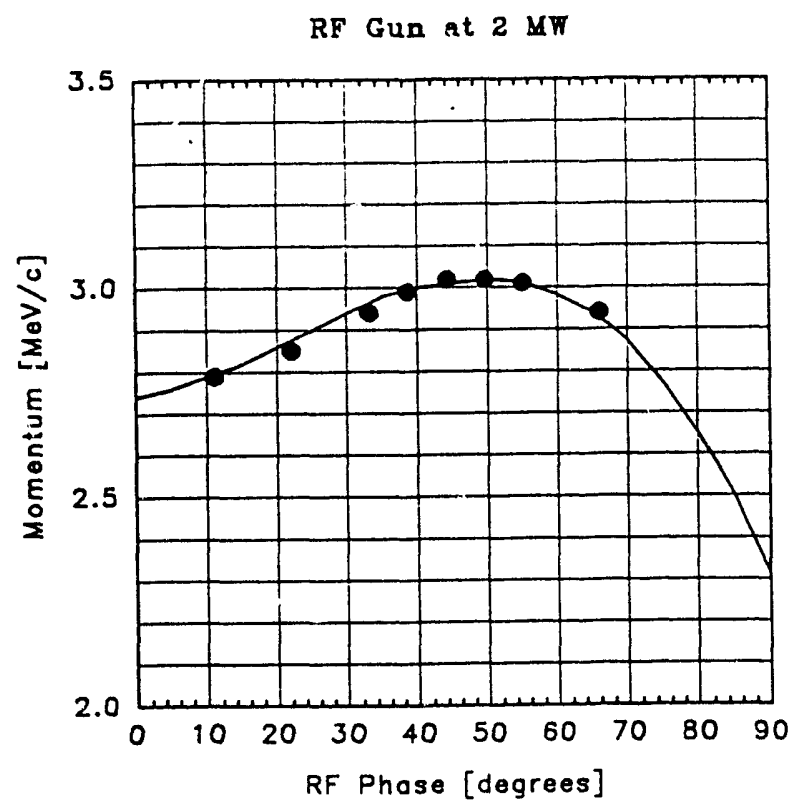

Fig. 6 The exit beam momentum as a function of the launch phase relative to the $\mathrm{rf}$ 

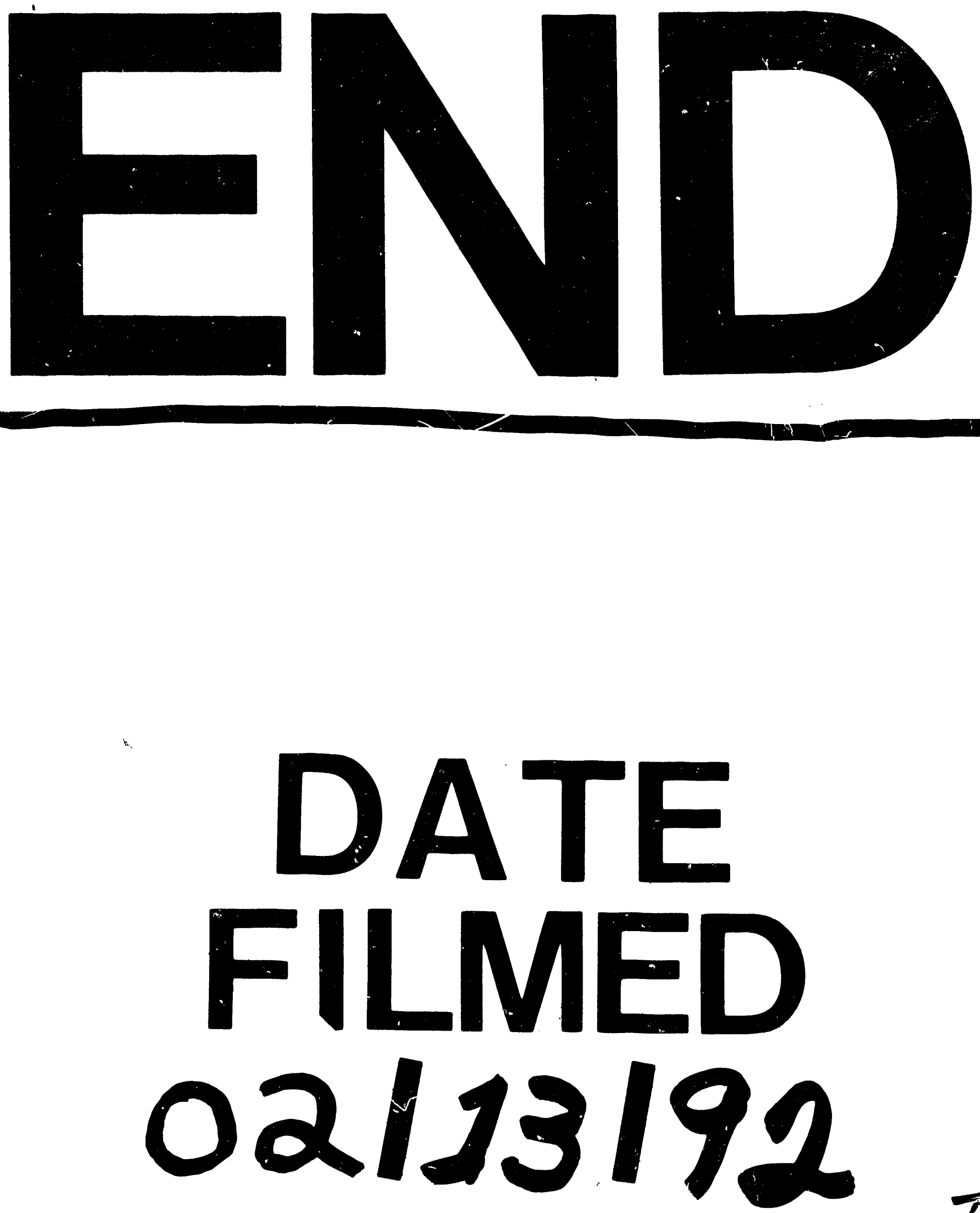
